\title{
An unusual finding of left ventriculography in Marfan syndrome with severe mitral valve prolapse
}

\author{
Masato Kimura ${ }^{1 *}$, Ryo Takahashi ${ }^{1}$, Eichiro Kawai ${ }^{1}$, Takehiko Ohnoki ${ }^{1}$, Osamu Adachi ${ }^{2}$, Yoshikatsu Saiki $^{2}$ and Shigeo Kure ${ }^{1}$
}

*Correspondence: mkimura774@med.tohoku.ac.jp

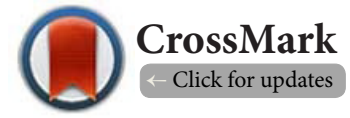

'Department of Pediatrics, Tohoku University School of Medicine, Sendai, Miyagi 980-8574, Japan.

${ }^{2}$ Division of Cardiovascular Surgery, Tohoku University School of Medicine, Sendai, Miyagi 980-8574, Japan.

\begin{abstract}
A 16-year-old boy with Marfan syndrome was scheduled for open-heart surgery for dilated aortic sinuses of Valsalva. Preoperative cardiac catheterization with left ventricular angiography demonstrated bulging at the junction of the mitral annulus with an appearance like 'a swim ring'. Transthoracic echocardiography showed severe mitral prolapse with the mitral valve leaflets rising toward the left atrium and stretching of the chordae tendineae. ECG-gated contrast CT revealed that this bulging was not the left ventricle itself but the interspace between the base of the left ventricle and mitral valve leaflets. This bulging disappeared after mitral valve plasty with an annuloplasty ring. This unusual finding might herald chordae tendinae rupture in Marfan syndrome.
\end{abstract}

Keywords: Marfan syndrome, mitral valve prolapse, left ventriculography, chordae tendinae rupture

\section{Introduction}

Marfan syndrome (MFS) is one of the most common connective tissue disorders, characterized by cardiovascular, skeletal and ocular abnormalities. Aortic root aneurysm and aortic dissection are the leading causes of morbidity and mortality in MFS [1]. Mitral valve prolapse, which is also a common cardiovascular complication of MFS, is considered to be due to myxomatous degeneration, redundant valve leaflets and long and/or thin chordae tendineae [2]. Although rupture of chordae tendineae is an uncommon complication of MFS, the consequences are serious [3-5]. To our knowledge, the signs of chordae tendinae rupture remain to be elucidated. This case describes an unusual finding of left ventriculography associated with severe mitral valve prolapse and rising mitral valve leaflets, which may be a sign of impeding chordae tendinae rupture in MFS.

\section{Case presentation}

A 16-year-old boy had a dilated aortic root due to MFS. His father and grandfather were also diagnosed with MFS and his father underwent an operation for aortic dissection in his thirties. The boy had cataract extraction for lens dislocation at the age of 6 and spinal fusion for scoliosis at the age of 12 . He was referred to our hospital at the age of 2 with dilated aortic sinuses. During outpatient follow-up, transthoracic echocardiography revealed dilated sinuses of Valsalva $(40 \mathrm{~mm},+2.5$ S.D.)
(Figure 1), normal-sized aortic annulus (23 $\mathrm{mm}$ ) and sinotubular junction $(27 \mathrm{~mm})$ and severe mitral valve prolapse with mild mitral regurgitation. The left ventricular long axis view also revealed a gap between the base of the left ventricle and posterior leaflet of the mitral valve in systole. Bicuspid aortic valve was also found. Cardiac angiography demonstrated a dilated aortic root with grade I aortic regurgitation. In addition, it showed bulging at the mitral annular junction in systole (Figure 2, Supplement video 1) with an appearance like 'a swim ring' in the right anterior oblique view and grade I mitral regurgitation. The ECG-gated cardiac computed tomography revealed that this bulging originated from the interspace between the base of the left ventricle and mitral valve leaflets, not the left ventricle itself. Mitral valve leaflets were rising toward the left atrium and there was displacement of the coaptation zone to the left atrium side (Figure 3, Supplement video 2). Bentall operation was carried out with a $21-\mathrm{mm}$ Regent ${ }^{\circledR}$ mechanical valve (St. Jude Medical, St Paul, MN) and 24-mm Gelweave graft conduit (Terumo CardioVascular Systems Corp., Ann Arbor, MI). Mitral valve plasty was performed with a 34-mm Carpentier-Edwards Physio II annuloplasty ring ${ }^{\circledR}$ (Edwards Lifesciences, Irvine, CA). Intraoperative inspection revealed that the mitral leaflets were not affected by myxomatous degeneration and the chordae tendineae did not appear to be thin and/or long. Severe mitral valve prolapse and rising of mitral valve leaflets toward the 


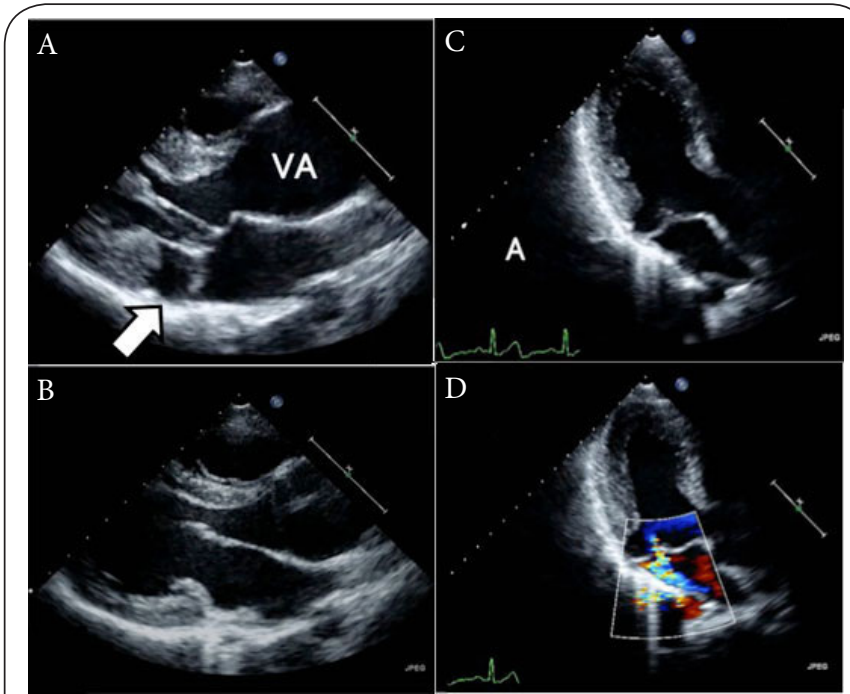

Figure 1. Transthoracic echocardiography.

(A) Dilated aortic sinuses of Valsalva (VA). (B) The interspace between the base of the left ventricle (arrow) and posterior leaflet of the mitral valve in systole. (C) The interspacedisappeared in diastole and (D) Severe mitral valve prolapse with mild mitral regurgitation.

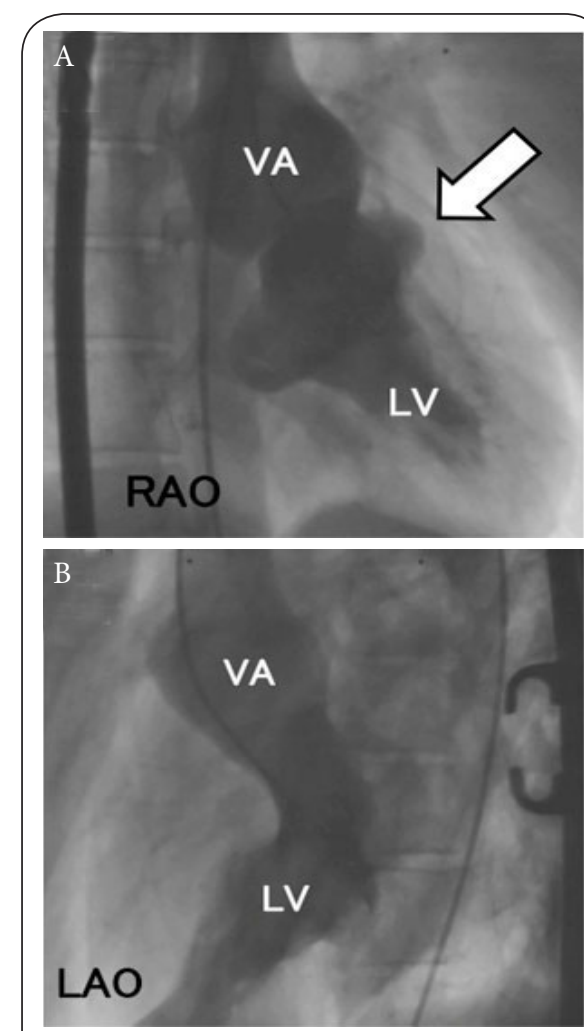

Figure 2. Left ventricular angiography showing the bulge at the junction of the mitral annulus (arrow) with grade I mitral regurgitation. (A) Right anterior oblique (RAO) view, (B) Left anterior oblique (LAO) view. LV: Left ventricle, VA: Aortic sinuses of valsalva.

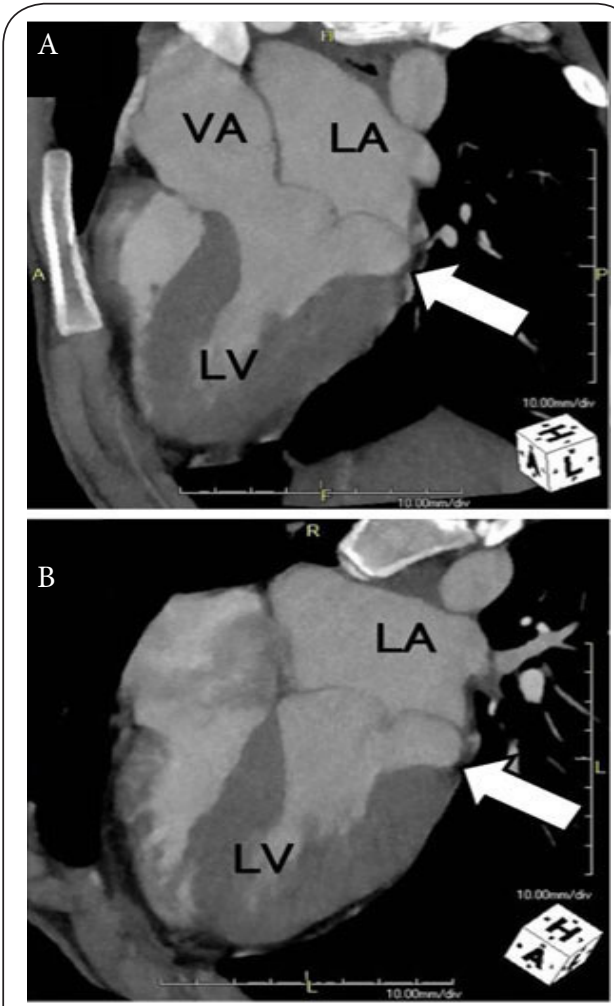

Figure 3. ECG-gated cardiac computed tomography (multiplanar reconstruction) showing the interspace between the base of the left ventricle and rising mitral valve leaflets (arrow). (A) Left ventricular longitudinal view, (B) Four chamber view. LV: Left ventricle, LA: Left atrium, VA: Aortic sinuses of valsalva.

left atrium resolved after annular reinforcement. He was discharged after an uneventful clinical course.

\section{Discussion and conclusion}

Marfan syndrome is a disorder of the connective tissues of the body, caused by mutations in the fibrillin-1 gene (FBN1) encoding for an extracellular glycoprotein called fibrillin [6]. Fibrillin is a major component of microfibrils that forms the scaffolding of elastic fibers. Although microfibrils are widely distributed throughout the body, they are particularly abundant in the aorta, ligaments and ciliary zonule of the lens that are prominently affected in MFS. Progressive dilatation and weakness (cystic medial necrosis) of the proximal aorta that might lead to rupture and sudden death is the most serious consequence of MFS [1]. Mitral valve prolapse, which is also a common cardiovascular complication of MFS, is considered to be due to myxomatous degeneration, redundant valve leaflets and long and/or thin chordae tendineae [2]. Mitral dysfunction in MFS is progressive with aging and about $80 \%$ of adult MFS patients have mitral regurgitation. In a series of 166 patients followed by Pyeritz and Wappel, 8 patients (4.8\%) died from mitral regurgitation or required mitral replacement 
[7]. Although rupture of chordae tendineae in MFS is rare complication, it leads to serious consequences such as severe heart failure acute surgical emergencies and death [3-5]. Gabbay and Tosefy reported in their review article [8] that the underlying causes of chordae tendinae rupture are mitral valve prolapse $(44.5 \%)$, subacute bacterial endocarditis (37.4\%), rheumatic heart disease (24.8\%), myxo-matous degeneration (11.7\%) and ischemic heart disease (1.4\%). Systemic connective tissue disorder such as MFS, osteogenesis imperfecta, and Ehler-Danlos syndrome are also causes. However, they did not mention or discuss the prelude of chordae tendinae rupture in their literature. In our case, the ECG-gated cardiac computed tomography revealed that the bulging seen on angiography was not the left ventricle itself but the gap between the base of the left ventricle and mitral valve leaflets (Figure 3). The presence of this interspace means loss of connection between the base of the left ventricle and mitral valve leaflets. In our experience, other MFS patients and non-MFS patients who have severe mitral prolapse do not have this sign, and the reason for is unknown. In echocardiography, this gap looks like a coronary sinus, especially a coronary sinus with a persistent left supra vena cava. Unlike the coronary sinus, this gap exists only in systole and exists just inside the pericardium. We speculate that this gap indirectly indicates excess tension of chordae tendineae, which may result in chordae tendinae dysfunction and/or rupture. The pathogenesis of mitral prolapse in MFS involves excess distensibility of the mitral annulus [9]. In our case, hyperflexibility of the mitral annulus is also an important factor in the pathogenesis of severe mitral prolapse because the interspace and mitral valve prolapse disappeared after reinforcement with an annuloplasty ring. Moreover, during intraoperative inspection, the anatomical structure of mitral leaflets, mitral annulus and chordae tendineae did not have degenerative changes and chordae tendineae were not long and thin.

In summary, we described a case with an unusual finding on left ventriculography in MFS with severe mitral valve prolapse. It might be an important sign of impeding chordae tendinae rupture in MFS and reinforcement of the mitral annulus should be considered.

\section{Additional files}

\section{Supplement video 1 \\ Supplement video 2}

\section{Competing interests}

The authors declare that they have no competing interests.

\section{Authors' contributions}

\begin{tabular}{|l|c|c|c|c|c|c|c|}
\hline Authors' contributions & MK & RT & EK & TO & OA & YS & SK \\
\hline Collection and/or assembly of data & -- & $\checkmark$ & $\checkmark$ & -- & -- & -- & -- \\
\hline Data analysis and interpretation & -- & -- & -- & $\checkmark$ & $\checkmark$ & -- & -- \\
\hline Writing the article & $\checkmark$ & -- & -- & -- & -- & -- & -- \\
\hline Critical revision of the article & -- & -- & -- & -- & -- & $\checkmark$ & -- \\
\hline Final approval of article & -- & -- & -- & -- & -- & -- & $\checkmark$ \\
\hline
\end{tabular}

\section{Acknowledgement}

We Thank Kei Takase, the consultant radiologist of Division of Diagnostic Radiology, Tohoku University School of Medicine, Japan.

Publication history

Editors: Efstathios Vassiliadis, University of Copenhagen, Denmark. Richard Lange, University of Texas Health Science Center, USA. EIC: William Clifford Roberts, Baylor University Medical Center, USA. Received: 19-Mar-2015 Final Revised: 19-Apr-2015

Accepted: 23-Apr-2015 Published: 29-Apr-2015

\section{References}

1. Murdoch JL, Walker BA, Halpern BL, Kuzma JW and McKusick VA. Life expectancy and causes of death in the Marfan syndrome. N Engl J Med. 1972; 286:804-8. | Article | PubMed

2. Van Karnebeek CD, Naeff MS, Mulder BJ, Hennekam RC and Offringa M. Natural history of cardiovascular manifestations in Marfan syndrome. Arch Dis Child. 2001; 84:129-37. | Article | PubMed Abstract | PubMed Full Text

3. Ohtsubo S, Sakurai J, Suda H, Ohteki H and Itoh T. [Marfan's syndrome with annulo-aortic ectasia and ruptured mitral chorda--a case report of combined composite valve graft replacement of the aortic root and mitral valve replacement]. Nihon Kyobu Geka Gakkai Zasshi. 1991; 39:2096-101. | Article I PubMed

4. Simpson JW, Nora JJ and McNamara DG. Marfan's syndrome and mitral valve disease: acute surgical emergencies. Am Heart J. 1969; 77:96-9. | Article | PubMed

5. Weidenbach M, Brenner R, Rantamaki T and Redel DA. Acute mitral regurgitation due to chordal rupture in a patient with neonatal Marfan syndrome caused by a deletion in exon 29 of the FBN1 gene. Pediatr Cardiol. 1999; 20:382-5. | Article | PubMed

6. Milewicz DM. Identification of defects in the fibrillin gene and protein in individuals with the Marfan syndrome and related disorders. Tex Heart Inst J. 1994; 21:22-9. | PubMed Abstract | PubMed Full Text

7. Pyeritz RE and Wappel MA. Mitral valve dysfunction in the Marfan syndrome. Am J Med. 1983; 74:797-807. | Article

8. Gabbay $U$ and Yosefy $C$. The underlying causes of chordae tendinae rupture: a systematic review. Int J Cardiol. 2010; 143:113-8. | Article | PubMed

9. Pini R, Roman MJ, Kramer-Fox R and Devereux RB. Mitral valve dimensions and motion in Marfan patients with and without mitral valve prolapse. Comparison to primary mitral valve prolapse and normal subjects. Circulation. 1989; 80:915-24. | Article | PubMed

\section{Citation}

Kimura M, Takahashi R, Kawai E, Ohnoki T, Adachi O, Saiki Y and Kure S. An unusual finding of left ventriculography in Marfan syndrome with severe mitral valve prolapse. Cardio Vasc Syst. 2015; 3:2. http://dx.doi.org/10.7243/2052-4358-3-2 